\title{
LOCAL ANALYSIS OF FRAME MULTIRESOLUTION ANALYSIS WITH A GENERAL DILATION MATRIX
}

\author{
Hong Oh Kim, Rae Young Kim and Jae Kun Lim
}

\begin{abstract}
A multivariate semi-orthogonal frame multiresolution analysis with a general integer dilation matrix and multiple scaling functions is considered. We first derive the formulas of the lengths of the initial (central) shift-invariant space $V_{0}$ and the next dilation space $V_{1}$, and, using these formulas, we then address the problem of the number of the elements of a wavelet set, that is, the length of the shift-invariant space $W_{0}:=V_{1} \ominus V_{0}$. Finally, we show that there does not exist a 'genuine' frame multiresolution analysis for which $V_{0}$ and $V_{1}$ are quasi-stable spaces satisfying the usual length condition.
\end{abstract}

\section{INTRODUCTION}

The orthonormal dyadic multiresolution analysis of $L^{2}(\mathbb{R})$ with a single scaling function was introduced by Mallat and Meyer in order to construct an orthonormal wavelet basis of $L^{2}(\mathbb{R})[17,18]$. Benedetto and Li considered the dyadic semi-orthogonal frame multiresolution analysis of $L^{2}(\mathbb{R})$ with a single scaling function, and successfully applied the theory in the analysis of narrow band signals [1]. We refer to [9] for the basic definitions and properties of frames and Riesz bases of a Hilbert space. Unlike the multiresolution analysis of Mallat and Meyer, where there always exists a wavelet set consisting of a single element whose dyadic dilations of the integer translates form an orthonormal basis of $L^{2}(\mathbb{R})$, the multiresolution analysis of Benedetto and $\mathrm{Li}$ has a wavelet set whose cardinality may be one or two [14]. The exact definition of a wavelet set of a multiresolution analysis is found in Section 3. The characterisation of the dyadic semi-orthogonal frame multiresolution analysis with a single scaling function admitting a single frame wavelet whose dyadic dilations of the integer translates form a frame for $L^{2}(\mathbb{R})$ was obtained, independently, by Benedetto and Treiber by a direct method [2], and by Kim and Lim by using the theory of shift-invariant spaces [14]. The dyadic multivariate generalisation (with a single scaling function) of the multiresolution analysis of Mallat and Meyer were considered by several authors. See [3], for example. Lim, among other things, addressed the problem of the cardinality of a wavelet set in the setting of

Received 10th September, 2002

The first and the second author were supported by KRF-2002-070-000004.

Copyright Clearance Centre, Inc. Serial-fee code: 0004-9727/03 \$A2.00+0.00. 
the dyadic multivariate frame multiresolution analysis with a single scaling function [16], and Kim and Lim gave an analysis of dyadic multivariate frame multiresolution analysis with multiple scaling functions [15]. As the theory and applications of wavelets evolve, many authors considered more general dilations other than the dyadic ones (for example, [8]).

In this paper we consider a multivariate frame multiresolution analysis with a general integer dilation matrix and multiple scaling functions by extending the method and results of [15]. We first analyse the local dimension of the initial, that is, central, shift-invariant space $V_{0}$ and the next dilation space $V_{1}$ of the multiresolution analysis. Using this, we derive the formulas of the lengths of the shift-invariant spaces $V_{0}$ and $V_{1}$, and address the problem of the number of the elements of a wavelet set. Finally, we show that there does not exist a 'genuine' frame multiresolution analysis for which $V_{0}$ and $V_{1}$ are quasi-stable spaces satisfying the usual length condition by applying the local dimension analysis and the ergodicity of the dilation matrix (Theorem 9). This result improves Theorem 3.9 in [15] in the sense that we do not presuppose that the spectrums of $V_{0}$ and $V_{1}$ coincide.

The organisation of this paper is as follows: Preliminary discussions on the dilation matrix and shift-invariant spaces and the definition of the multiresolution analysis we consider are given in Section 2, and our main results, along with an analysis of the local dimensions of $V_{0}$ and $V_{1}$, are given in Section 3.

\section{PRELIMINARY Discussion}

Suppose that $M$ is a $d \times d$ integer dilation matrix, that is, the entries of $M$ are integers and the moduli of the eigenvalues of $M$ are strictly greater than one. It is known that the order of the quotient group $\mathbb{Z}^{d} / M \mathbb{Z}^{d}$ is $|\operatorname{det} M|$ [8, Lemma 2]. Let $\mathbb{T}^{d}:=\mathbb{R}^{d} / \mathbb{Z}^{d}$ denote the $d$-dimensional torus which is identified with $[-1 / 2,1 / 2)^{d}$. For $x \in \mathbb{R}^{d}$, let $x(\bmod 1)$ denotes the standard representative of $x+\mathbb{Z}^{d}$ in $[-1 / 2,1 / 2)^{d}$. Suppose that $T$ is a $d \times d$ invertible matrix with integer entries such that the moduli of the eigenvalues of $T$ are all different from 1 . Then the map $\widetilde{T}: \mathbb{T}^{d} \rightarrow \mathbb{T}^{d}$, defined via $\widetilde{T} x:=T x(\bmod 1)$, is ergodic [20, Theorem 0.15 , Corollary 1.10 .1$]$. We note that $M^{t}=M^{*}$, where $t$ and $*$ denote the transpose and the adjoint of a matrix with complex entries, respectively. For notational convenience we let $Q:=\mathbb{Z}^{d} / M \mathbb{Z}^{d}$ and let $Q^{*}:=\mathbb{Z}^{d} / M^{*} \mathbb{Z}^{d}$. Let $D:=D_{M}: L^{2}\left(\mathbb{R}^{d}\right) \rightarrow L^{2}\left(\mathbb{R}^{d}\right)$ denote the unitary dilation operator defined via $D f(x):=|\operatorname{det} M|^{1 / 2} f(M x)$. For $y \in \mathbb{R}^{d}, T_{y}: L^{2}\left(\mathbb{R}^{d}\right)$ $\rightarrow L^{2}\left(\mathbb{R}^{d}\right)$ denotes the unitary translation operator such that $T_{y} f(x):=f(x-y)$. In this paper we adapt the following definition of multiresolution analysis.

DEFINITION 1: $\left\{V_{k}\right\}_{k \in Z}$ is said to be a frame multiresolution analysis if each $V_{k}$ is a closed subspace of $L^{2}\left(\mathbb{R}^{d}\right)$ such that: 
(1) $V_{k} \subset V_{k+1}, \quad k \in \mathbb{Z}$;

(2) $\overline{\bigcup_{k \in \mathbf{Z}} V_{k}}=L^{2}\left(\mathbb{R}^{d}\right), \bigcap_{k \in \mathbf{Z}} V_{k}=\{0\}$;

(3) $D\left(V_{k}\right)=V_{k+1}, \quad k \in \mathbb{Z}$;

(4) There exists a finite set of scaling functions $\Phi \subset V_{0}$ such that $\left\{T_{\alpha} \varphi: \alpha\right.$ $\left.\in \mathbb{Z}^{d}, \varphi \in \Phi\right\}$ is a frame for $V_{0}$.

Various examples and applications of multiresolution analyses are found in the references cited in Section 1.

The following form of the Fourier transform is used throughout this paper: for $f \in L^{1}\left(\mathbb{R}^{d}\right) \cap L^{2}\left(\mathbb{R}^{d}\right)$ and $t \in \mathbb{R}^{d} \widehat{f}(t):=\int_{\mathbb{R}^{d}} f(x) e^{-2 \pi i t \cdot x} d x$, where $\cdot$ denotes the $d$ dimensional real inner product. It is, of course, extended to be a unitary transform from $L^{2}\left(\mathbb{R}^{d}\right)$ onto $L^{2}\left(\mathbb{R}^{d}\right)$ via the Plancherel theorem.

Suppose that $\left\{f_{i}: 1 \leqslant i \leqslant n\right\}$ is a finite family of elements of a Hilbert space $\mathcal{H}$ with an inner product $\langle\cdot, \cdot\rangle$. We frequently use the following simple observation: $\operatorname{dim} \operatorname{span}\left\{f_{i}: 1 \leqslant i \leqslant n\right\}=\operatorname{rank}\left(\left\langle f_{i}, f_{j}\right\rangle\right)_{1 \leqslant i, j \leqslant n}$.

Our analysis uses the theory of (multi-integer) shift-invariant spaces developed in $[4,5,6,10,11,12,19]$ and the references therein. We briefly review the theory and uses the existing results freely. A closed subspace $S$ of $L^{2}\left(\mathbb{R}^{d}\right)$ is said to be shift-invariant if $T_{\alpha} f \in S$ whenever $f \in S$ and $\alpha \in \mathbb{Z}^{d}$. If $\Phi \subset L^{2}\left(\mathbb{R}^{d}\right)$, then $S:=\mathcal{S}(\Phi):=\overline{\operatorname{span}}\left\{T_{\alpha} \varphi: \alpha\right.$ $\left.\in \mathbb{Z}^{d}\right\}$ is a shift-invariant space. In this case, $\Phi$ is called a generator of $S$. If $\Phi$ is finite, then $S$ is called a finite shift-invariant space. We write $S=\mathcal{S}(\varphi)$ instead of $\mathcal{S}(\{\varphi\})$ if $\Phi=\{\varphi\}$ is a singleton. In this case, we call $S$ a principal shift-invariant space. It is known that any shift-invariant space has a countable generator. The length of a shift-invariant space is defined to be

$$
\operatorname{len} S:=\inf \left\{\# \Phi: S=\mathcal{S}(\Phi), \Phi \subset L^{2}\left(\mathbb{R}^{d}\right)\right\}
$$

where \# denotes the cardinality. Let $\widehat{f}_{\| x}$ be the sequence $(\hat{f}(x+\alpha))_{\alpha \in \mathbf{Z}^{d}}$ which is in $\ell^{2}\left(\mathbb{Z}^{d}\right)$ for almost every $x \in \mathbb{T}^{d}$. If $A \subset L^{2}\left(\mathbb{R}^{d}\right), x \in \mathbb{T}^{d}$, then we let $\widehat{A}_{\| x}:=\left\{\widehat{f}_{\| x} \in \ell^{2}\left(\mathbb{Z}^{d}\right): f\right.$ $\in A\}$, which is called the fibre of $A$ at $x$. It is a subspace of $\ell^{2}\left(\mathbb{Z}^{d}\right)$ if $A$ is a shift-invariant space. The following theorem is used frequently in our discussion.

THEOREM 2. ([4, 6, 10, 11].) Let $S$ be a closed, not necessarily shift-invariant, subspace of $L^{2}\left(\mathbb{R}^{d}\right)$ and $\Phi$ a countable subset of $L^{2}\left(\mathbb{R}^{d}\right)$. Then $S=\mathcal{S}(\Phi)$ if and only if $\widehat{f}_{\| x} \in \overline{\operatorname{span}}\left\{\widehat{\varphi}_{\| x}: \varphi \in \Phi\right\}$ for almost every $x \in \mathbb{T}^{d}$ and for each $f \in S$.

The spectrum of a shift-invariant space is defined to be $\sigma(S):=\left\{x \in \mathbb{T}^{d}: \widehat{S}_{\| x} \neq\{0\}\right\}$. A finite subset $\Phi$ of $L^{2}\left(\mathbb{R}^{d}\right)$ is said to be a quasi-stable generator for the shift-invariant space $\mathcal{S}(\Phi)$ if, in addition to the condition that the family of the integer translates of $\Phi$ is a frame for $\mathcal{S}(\Phi), \operatorname{dim} \operatorname{span}\left\{\widehat{\varphi}_{\| x}: \varphi \in \Phi\right\}=\# \Phi$ or 0 for almost every $x \in \mathbb{T}^{d}$. If $\Phi$ is a quasi-stable generator, then there is a convenient 'local' formula for the orthogonal projection onto $\mathcal{S}(\Phi)[4, \mathbf{1 9}]$. The stable generator is a quasi-stable generator such that 
the spectrum of the shift-invariant space it generates is $\mathbb{T}^{d}$. It turns out that if $\Phi$ is a stable generator, then the family of the integer translates of $\Phi$ is a Riesz basis for $\mathcal{S}(\Phi)$ $[4,19]$. We say that a shift-invariant space $S$ is quasi-stable, if $\operatorname{dim} \widehat{S}_{\| x}=n$ or 0 for some non-negative integer $n$ almost everywhere. It is said to be stable if $\operatorname{dim} \widehat{S}_{|| x}=n$ almost everywhere. It is known that a quasi-stable/stable shift-invariant space has a quasi-stable/stable generator $[4,19]$.

We need the following results:

THEOREM 3. ([4].) For a shift-invariant subspace $S$ of $L^{2}\left(\mathbb{R}^{d}\right)$

$$
\operatorname{len} S=\operatorname{ess-sup}\left\{\operatorname{dim} \widehat{S}_{\| x}: x \in \mathbb{T}^{d}\right\} \text {. }
$$

THEOREM 4. ([4].) Let $S_{1}$ be a shift-invariant subspace of a shift-invariant space $S$ and let $S_{2}:=S \ominus S_{1}$. Then $S_{2}$ is also a shift-invariant subspace of $S$ and $\widehat{S}_{\| x}$ $=\widehat{S}_{1 \mid x} \oplus \widehat{S}_{2 \| x}$ for almost every $x \in \mathbb{T}^{d}$.

Suppose that $S=\mathcal{S}(\Phi)$ for a finite set $\Phi:=\left\{\varphi_{1}, \varphi_{2}, \ldots, \varphi_{n}\right\}$. Then the $n \times n$ matrix

$$
G(x):=G_{\Phi}(x):=\left(\left\langle\widehat{\varphi}_{i|| x}, \widehat{\varphi_{j}}\right\rangle_{\ell^{2}\left(\mathbf{Z}^{d}\right)}\right)_{1 \leqslant i, j \leqslant n}
$$

is the Gramian of $\Phi$ at $x \in \mathbb{T}^{d}$. Let $\lambda(x), \lambda^{+}(x)$ and $\Lambda(x)$ denote the smallest eigenvalue, the smallest non-negative eigenvalue and the largest eigenvalue of $G(x)$, respectively.

ThEOREM 5. ([4, 6, 19].) The family of the integer translates of $\Phi$ is a frame for $S$ if and only if there exist positive constants $A$ and $B$ such that $A \leqslant \lambda^{+}(x) \leqslant \Lambda(x)$ $\leqslant B$ for almost every $x \in \sigma(S)$. It is a Riesz basis for $S$ if and only if $A \leqslant \lambda(x) \leqslant \Lambda(x)$ $\leqslant B$ for almost every $x \in \mathbb{T}^{d}$. Moreover, $A$ and $B$ are a pair of frame (Riesz) bounds of the frame (Riesz basis), respectively.

\section{Frame multiresolution anALySiS}

Suppose that $\left\{V_{j}\right\}_{j \in \mathbf{Z}}$ is a frame multiresolution analysis. Then there exists a set of scaling functions $\Phi:=\left\{\varphi_{i}: 1 \leqslant i \leqslant n\right\} \subset L^{2}\left(\mathbb{R}^{d}\right)$ such that $\left\{T_{\alpha} \varphi_{i}: \alpha \in \mathbb{Z}^{d}, 1 \leqslant i \leqslant n\right\}$ is a frame for $V_{0}$. We may assume that the length of $V_{0}$ is $n$. Then $V_{0}=\mathcal{S}(\Phi)$ and $V_{1}:=D\left(V_{0}\right)$. Let

be the Gramian of $\Phi$ at $x \in \mathbb{T}^{d}$.

$$
G(x):=G_{\Phi}(x):=\left(\left\langle\widehat{\varphi}_{i|| x}, \widehat{\varphi_{j}}\right\rangle_{\ell^{2}\left(\mathbf{Z}^{d}\right)}\right)_{1 \leqslant i, j \leqslant n}
$$

Since $\varphi_{i} \in V_{1}$ for each $1 \leqslant i \leqslant n$, and since $\left\{D T_{\alpha} \varphi_{i}: \alpha \in \mathbb{Z}^{d}, 1 \leqslant i \leqslant n\right\}$ is a frame for $V_{1}$, there exist $a_{i j} \in \ell^{2}\left(\mathbb{Z}^{d}\right), 1 \leqslant i, j \leqslant n$, such that $\varphi_{i}=\sum_{j=1}^{n} \sum_{\alpha \in \mathbf{Z}^{d}} a_{i j}(\alpha) D T_{\alpha} \varphi_{j}$. Hence

$$
\begin{aligned}
\widehat{\varphi}_{i}(x) & =\sum_{j=1}^{n} \sum_{\alpha \in \mathbf{Z}^{d}} a_{i j}(\alpha)|\operatorname{det} M|^{-1 / 2} e^{-2 \pi i \alpha \cdot\left(M^{*-1} x\right)} \widehat{\varphi}_{j}\left(M^{*-1} x\right) \\
& =\sum_{j=1}^{n} m_{i j}\left(M^{*-1} x\right) \widehat{\varphi}_{j}\left(M^{*-1} x\right)
\end{aligned}
$$


where

$$
m_{i j}(x):=\sum_{\alpha \in \mathbf{Z}^{d}}|\operatorname{det} M|^{-1 / 2} a_{i j}(\alpha) e^{-2 \pi i \alpha \cdot x} \in L^{2}\left(\mathbb{T}^{d}\right)
$$

For $x \in \mathbb{T}^{d}$, let

$$
m(x):=\left(m_{i j}(x)\right)_{1 \leqslant i, j \leqslant n}
$$

and

$$
\widehat{\Phi}(x):=\left(\widehat{\varphi}_{1}(x), \widehat{\varphi}_{2}(x), \ldots, \widehat{\varphi}_{n}(x)\right)^{t}
$$

Then

$$
\widehat{\Phi}(x)=m\left(M^{*-1} x\right) \widehat{\Phi}\left(M^{*-1} x\right)
$$

This $m$, called a mask of the multiresolution analysis, may not be unique since $\left\{D T_{\alpha} \varphi_{i}\right.$ : $\left.\alpha \in \mathbb{Z}^{d}, 1 \leqslant i \leqslant n\right\}$ is only assumed to be a frame, not necessarily a Riesz basis. Note that $D T_{M y}=T_{y} D$ for $y \in \mathbb{R}^{d}$. Since each $\gamma \in \mathbb{Z}^{d}$ can be written uniquely as $\gamma=M \alpha+\beta$ for some $\alpha \in \mathbb{Z}^{d}$ and $\beta \in Q$,

$$
\left\{D T_{\gamma} \varphi_{i}: \gamma \in \mathbb{Z}^{d}, 1 \leqslant i \leqslant n\right\}=\left\{T_{\alpha} D T_{\beta} \varphi_{i}: \alpha \in \mathbb{Z}^{d}, \beta \in Q, 1 \leqslant i \leqslant n\right\}
$$

Hence $V_{1}=\mathcal{S}(\Pi)$, where

$$
\Pi:=\left\{D T_{\beta} \varphi_{i}: \beta \in Q, 1 \leqslant i \leqslant n\right\}
$$

This implies that the length of the shift-invariant space $V_{1}$ is less than or equal to $n|\operatorname{det} M|$. Since $V_{0}$ is a shift-invariant subspace of $V_{1}$, len $V_{1} \geqslant \operatorname{len} V_{0}=n$. There is an example of a frame multiresolution analysis in which the length of $V_{1}$ is that of $V_{0}$. See Example 6 below. Let $W_{0}$ denote $V_{1} \ominus V_{0}$, and let $W_{j}:=D^{j}\left(W_{0}\right), j \in \mathbb{Z}$. Then Definition 1 implies that $L^{2}\left(\mathbb{R}^{d}\right)=\bigoplus_{j \in \mathbf{Z}} W_{j}$. $W_{0}$ is a shift-invariant space by Theorem 4 . Since $W_{0}$ is a subspace of $V_{1}$, the length of $W_{0}$ is also less than or equal to $n|\operatorname{det} M|$. It cannot be zero. If it were zero, then $W_{0}=\{0\}$; hence $V_{0}=V_{1}$. Definition 1 implies that $L^{2}(\mathbb{R})=V_{0}=\mathcal{S}(\Phi)$. This contradicts a result in [7] which states roughly that there are no frames of $L^{2}\left(\mathbb{R}^{d}\right)$ consisting of the translates of a finite number of functions. Since $W_{0}$ is a finite shift-invariant space, there is a finite set $\Psi$, called a wavelet set, such that $W_{0}=\mathcal{S}(\Psi)$. We may assume that the integer translates of the elements of $\Psi$ form a frame for $W_{0}[5,19]$. Then, obviously, $\left\{D^{j} T_{\alpha} \psi: j \in \mathbb{Z}, \alpha \in \mathbb{Z}^{d}, \psi \in \Psi\right\}$ is a frame for $L^{2}\left(\mathbb{R}^{d}\right)$. Since the minimal cardinality of such $\Psi$ is len $W_{0}$, the (minimal) number of the elements of a wavelet set is len $W_{0}$.

Note that, for $\beta \in \mathbb{Z}^{d}$ and $x \in \mathbb{T}^{d}$,

$$
\begin{aligned}
\left(D T_{\beta} \varphi_{i}\right)^{\wedge}(x) & =|\operatorname{det} M|^{-1 / 2} e^{-2 \pi i \beta \cdot\left(M^{*-1} x\right)} \widehat{\varphi}_{i}\left(M^{*-1} x\right) \\
\left(D T_{\beta} \varphi_{i}\right)_{\| x} & =|\operatorname{det} M|^{-1 / 2} e^{-2 \pi i \beta \cdot\left(M^{*-1} x\right)}\left(e^{-2 \pi i \beta \cdot\left(M^{*-1} \alpha\right)} \widehat{\varphi}_{i}\left(M^{*-1}(x+\alpha)\right)\right)_{\alpha \in \mathbf{Z}^{d}}
\end{aligned}
$$


Hence, for almost every $x \in \mathbb{T}^{d}$,

$$
\begin{aligned}
\widehat{V}_{1 \| x} & =\operatorname{span}\left\{\left(e^{-2 \pi i \beta \cdot\left(M^{*-1} \alpha\right)} \widehat{\varphi}_{i}\left(M^{*-1}(x+\alpha)\right)\right)_{\alpha \in \mathbf{Z}^{d}}: \beta \in Q, 1 \leqslant i \leqslant n\right\}, \\
\widehat{V}_{0 \| x} & =\operatorname{span}\left\{(\widehat{\varphi}(x+\alpha))_{\alpha \in \mathbf{Z}^{d}}: 1 \leqslant i \leqslant n\right\} \\
& =\operatorname{span}\left\{\sum_{j=1}^{n}\left(m_{i j}\left(M^{*-1}(x+\alpha)\right) \widehat{\varphi}_{j}\left(M^{*-1}(x+\alpha)\right)\right)_{\alpha \in \mathbf{Z}^{d}}: 1 \leqslant i \leqslant n\right\} .
\end{aligned}
$$

For $\beta^{*} \in Q^{*}$ define $P_{\beta^{*}}: \ell^{2}\left(\mathbb{Z}^{d}\right) \rightarrow \ell^{2}\left(\mathbb{Z}^{d}\right)$ via

$$
\left(P_{\beta} \cdot a\right)(\alpha):= \begin{cases}a(\alpha), & \text { if } \alpha \in \beta^{*}+M^{*} \mathbb{Z}^{d}, \\ 0, & \text { otherwise. }\end{cases}
$$

Then $\ell^{2}\left(\mathbb{Z}^{d}\right)=\bigoplus_{\beta^{*} \in Q^{*}} P_{\beta^{*}}\left(\ell^{2}\left(\mathbb{Z}^{d}\right)\right)$. Define, for $x \in \mathbb{T}^{d}, 1 \leqslant i \leqslant n, \beta^{*} \in Q^{*}$,

$$
a_{x, i, \beta^{*}}:=P_{\beta^{*}}\left(\left(\widehat{\varphi}_{i}\left(M^{*-1}(x+\alpha)\right)\right)_{\alpha \in \mathbf{Z}^{d}}\right) .
$$

Notice that $a_{x, i, \beta^{*}}$ is the 'up-sampled' version of $\widehat{\varphi}_{i \| M^{*-1}\left(x+\beta^{*}\right)}$, that is,

$$
\begin{aligned}
a_{x, i, \beta^{*}}\left(\beta^{*}+M^{*} \alpha\right) & =\widehat{\varphi}_{i \| M^{*-1}\left(x+\beta^{*}\right)}(\alpha), \quad \alpha \in \mathbb{Z}^{d}, \\
a_{x, i, \beta^{*}}\left(\gamma^{*}\right) & =0, \quad \gamma^{*} \notin \beta^{*}+M^{*} \mathbb{Z}^{d} .
\end{aligned}
$$

Therefore

$$
\left\|a_{x, i, \beta^{*}}\right\|_{\ell^{2}(\mathbf{Z})}=\left\|\widehat{\varphi}_{i \| M^{*-1}\left(x+\beta^{*}\right)}\right\|_{\ell^{2}(\mathbf{Z})}
$$

We also have

$$
\left(e^{-2 \pi i \beta \cdot\left(M^{*-1} \alpha\right)} \widehat{\varphi}_{i}\left(M^{*-1}(x+\alpha)\right)\right)_{\alpha \in \mathbf{Z}}=\sum_{\gamma^{*} \in Q^{*}} e^{-2 \pi i \beta \cdot\left(M^{*-1} \gamma^{*}\right)} a_{x, i, \gamma^{*}}
$$

Let $b_{x, i, \beta}$ be the right-hand side of the above equation. Then, for a fixed $x \in \mathbb{T}^{d}, 1, \leqslant i \leqslant n$, we have the following matrix relation:

$$
\left(b_{x, i, \beta}\right)_{\beta \in Q}^{t}=\left(e^{-2 \pi i \beta \cdot\left(M^{*-1} \gamma^{*}\right)}\right)_{\beta \in Q, \gamma^{*} \in Q^{*}}\left(a_{x, i, \gamma^{*}}\right)_{\gamma^{*} \in Q^{*}}^{t}
$$

Recall that, for any $\beta \in \mathbb{Z}^{d}$, the map $\gamma^{*} \rightarrow e^{-2 \pi i \beta \cdot\left(M^{*-1} \gamma^{*}\right)}$ is a character of the discrete group $G^{*}$. Hence the sum $\sum_{\gamma^{*} \in Q^{*}} e^{-2 \pi i \beta \cdot\left(M^{*-1} \gamma^{*}\right)}$ is the order of $Q^{*}$, which is $|\operatorname{det} M|$, if the map is the identity character, and the sum is 0 if the map is not the identity character since the only discrete multiplicative subgroups of $\mathbb{T}$ are the groups of the $p$-th roots of unity ([20, Theorem 0.14]). Using this observation, it is easy to see that

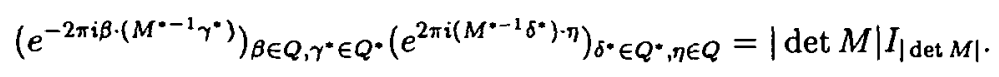


In particular, for each $1 \leqslant i \leqslant n$ and $x \in \mathbb{T}^{d}, \operatorname{span}\left\{b_{x, i, \beta}: \beta \in Q\right\}=\operatorname{span}\left\{a_{x, i, \gamma^{*}}: \gamma^{*}\right.$ $\left.\in Q^{*}\right\}$. This shows that:

$$
\widehat{V}_{1|| x}=\operatorname{span}\left\{a_{x, i, \gamma^{*}}: 1 \leqslant i \leqslant n, \gamma^{*} \in Q^{*}\right\} .
$$

The 1-periodicity of the mask $m$ and (4) imply that:

$$
\widehat{V}_{0 \| x}=\operatorname{span}\left\{\sum_{j=1}^{n} \sum_{\alpha^{*} \in Q^{*}} m_{i j}\left(M^{*-1}\left(x+\alpha^{*}\right)\right) a_{x, j, \alpha^{*}}: 1 \leqslant i \leqslant n\right\} .
$$

Note that, for almost every $x \in \mathbb{T}^{d}, \operatorname{dim} \widehat{V}_{1|| x}$ equals the rank of the following $n|\operatorname{det} M|$ $\times n|\operatorname{det} M|$ matrix

$$
\left(\left\langle a_{x, i, \alpha^{*}}, a_{x, j, \beta^{*}}\right\rangle_{\mathbf{C}^{d}}\right)_{\left(i, \alpha^{*}\right),\left(j, \beta^{*}\right)} .
$$

If we order the indices suitably, then (4) and (5) imply that the matrix is the block diagonal matrix

$$
\operatorname{diag}\left(G\left(M^{*-1}\left(x+\alpha^{*}\right)\right)\right)_{\alpha^{*} \in Q^{*}} .
$$

Recall that $\operatorname{rank} G\left(M^{*-1}\left(x+\alpha^{*}\right)\right)=\operatorname{dim} \widehat{V}_{0|| M^{*-1}\left(x+\alpha^{*}\right)}$ for each $\alpha^{*} \in Q^{*}$. Hence, for almost every $x \in \mathbb{T}^{d}$,

$$
\operatorname{dim} \widehat{V}_{1 \| x}=\sum_{\alpha^{*} \in Q^{*}} \operatorname{rank} G\left(M^{*-1}\left(x+\alpha^{*}\right)\right)=\sum_{\alpha^{*} \in Q^{*}} \operatorname{dim} \widehat{V}_{0 \| M^{*-1}\left(x+\alpha^{*}\right)}
$$

A direct calculation shows that

$$
G(x)=\sum_{\alpha^{*} \in Q^{*}} m\left(M^{*-1}\left(x+\alpha^{*}\right)\right) G\left(M^{*-1}\left(x+\alpha^{*}\right)\right) m\left(M^{*-1}\left(x+\alpha^{*}\right)\right)^{*} .
$$

Hence, for almost every $x \in \mathbb{T}^{d}$,

$$
\operatorname{dim} \widehat{V}_{0 \| x}=\operatorname{rank} \sum_{\alpha^{*} \in Q^{*}} m\left(M^{*-1}\left(x+\alpha^{*}\right)\right) G\left(M^{*-1}\left(x+\alpha^{*}\right)\right) m\left(M^{*-1}\left(x+\alpha^{*}\right)\right)^{*} .
$$

EXAMPLE 6. Let us first consider a dyadic univariate frame multiresolution analysis with a single scaling function, that is, $d=n=1$ and $Q=\mathbb{Z} / 2 \mathbb{Z}=\{0,1\}$. Therefore $D f(x)=2^{1 / 2} f(2 x)$. Let $V_{0}$ be a Paley-Wiener space such that $\left\{f \in L^{2}(\mathbb{R}): \operatorname{supp}(f)\right.$ $\subset[-a, a]\}$ with $0<a<1 / 4$, and let $V_{j}:=D^{j}\left(V_{0}\right), j \in \mathbb{Z}$. Then it is easy to see that $\left\{V_{j}\right\}_{j \in \mathbf{Z}}$ is a frame multiresolution analysis ([14]). Obviously, $V_{0}=\mathcal{S}\left(\check{\chi}_{[-a, a]}\right)$, where $\vee$ denotes the inverse Fourier transform. Hence, $V_{1}=\mathcal{S}\left(\check{\chi}_{[-2 a, 2 a]}\right)$ is a shift-invariant space of length 1. This can be proved by using (8). Note that $G(x)=\chi_{[-a, a]+\mathbf{z}}(x)$ for $x \in \mathbb{T}$. Hence, $\widehat{V}_{1 \| x}=\chi_{[-a, a]+\mathbf{Z}}(x / 2)+\chi_{[-a, a]+\mathbf{Z}}(x / 2+1 / 2)$ for $x \in \mathbb{T}$. Hence $\operatorname{dim} \widehat{V}_{1 \| x}=1$ for $x \in[-2 a, 2 a]$, and $\operatorname{dim} \widehat{V}_{1 \| x}=0$ for $x \in \mathbb{T} \backslash[-2 a, 2 a]$. Therefore len $V_{1}=1$ by Theorem 3 . Recall that len $V_{1}$ is less than or equal to $n 2^{d}=2$. In this example, the length of $V_{1}$ is 
that of $V_{0}$. Notice, however, that $V_{0}$ is a strict subspace of $V_{1}$. It is now easy to see that len $V_{2}=2$.

The above example can be directly extended to the case where $d>1, n=1$ and $M=2 I_{d}$. Then $Q=Q^{*}=\left\{\left(\varepsilon_{1}, \varepsilon_{2}, \ldots, \varepsilon_{d}\right): \varepsilon_{i}=0\right.$ or 1$\}$. For the sake of simplicity, define $\widehat{\varphi}:=\chi_{(-1 / 4,1 / 4)^{d}}, V_{0}^{d}:=\mathcal{S}(\varphi)$ and $V_{j}^{d}:=D^{j}\left(V_{0}^{d}\right)$. Then $\left\{V_{j}^{d}\right\}_{j \in \mathbf{Z}}$ is a frame multiresolution analysis. We show that $\operatorname{len} V_{1}^{d}=1$. (8) implies that, for almost every $x \in \mathbb{T}^{d}=[-1 / 2,1 / 2)^{d}, \operatorname{dim} \widehat{V_{1}^{d} \| x}$ is the number of the sets $2 \sigma\left(V_{0}^{d}\right)-\beta^{*}+2 \mathbb{Z}^{d}, \beta^{*} \in Q^{*}$, to which $x$ belongs. A direct calculation shows that $x$ belongs only to $2 \sigma\left(V_{0}^{d}\right)+2 \mathbb{Z}^{d}$ since $\sigma\left(V_{0}^{d}\right)=(-1 / 4,1 / 4)^{d}$. Therefore len $V_{1}^{d}=1$ by Theorem 3 . Since $V_{0}^{d}$ is a strict subset of $V_{1}^{d}$, len $W_{0}=1$.

Suppose, temporarily, that $\left\{T_{\alpha} \varphi_{i}: \alpha \in \mathbb{Z}, 1 \leqslant i \leqslant n\right\}$ is a Riesz basis of $V_{0}$. Then $\operatorname{rank} G(x)=n$ for almost every $x \in \mathbb{T}^{d}[4]$. Hence $\operatorname{dim} \widehat{V}_{1 \| x}=n|\operatorname{det} M|$ for almost every $x \in \mathbb{T}^{d}$. Since $\widehat{V}_{1 \| x}=\widehat{V}_{0 \| x} \oplus \widehat{W}_{0 \| x}$ almost everywhere by Theorem $4, \operatorname{dim} \widehat{W}_{0 \| x}$ $=n(|\operatorname{det} M|-1)$ almost everywhere. Therefore len $W_{0}=n(|\operatorname{det} M|-1)$ by Theorem 3 . Benedetto and $\mathrm{Li}[1]$ introduced the following concept: a frame multiresolution analysis admit a standard (frame) wavelet set if len $W_{0} \leqslant n(|\operatorname{det} M|-1)$. The characterisations of dyadic frame multiresolution analyses admitting standard wavelet sets were given in $[2,14]$, independently, for $d=n=1$, and in [16] for $d>1$ and $n=1$. Combining (8) and (9) with Theorems 3 and 4 yield the following general result on the admittance of a standard wavelet set.

THEOREM 7. The frame multiresolution analysis $\left\{V_{j}\right\}_{j \in \mathbf{Z}}$ admits a standard wavelet set if and only if, for almost every $x \in \mathbb{T}^{d}$,

$$
\begin{aligned}
& \sum_{\alpha^{*} \in Q^{*}} \operatorname{rank} G\left(M^{*-1}\left(x+\alpha^{*}\right)\right) \\
& \quad-\operatorname{rank} \sum_{\alpha^{*} \in Q^{*}} m\left(M^{*-1}\left(x+\alpha^{*}\right)\right) G\left(M^{*-1}\left(x+\alpha^{*}\right)\right) m\left(M^{*-1}\left(x+\alpha^{*}\right)\right)^{*} \\
& \quad \leqslant n(|\operatorname{det} M|-1) .
\end{aligned}
$$

We now recover the previous results on the admittance of a standard wavelet set $[2,14$, 16]. Suppose that $n=1$ and $M=2 I_{d}$. Then $Q=Q^{*}=\mathbb{Z}^{d} / 2 \mathbb{Z}^{d}=\left\{\left(\varepsilon_{1}, \varepsilon_{2}, \ldots, \varepsilon_{d}\right): \varepsilon_{i}\right.$ $=0$ or 1$\},|\operatorname{det} M|=2^{d}, \Phi=\{\varphi\} \subset L^{2}\left(\mathbb{R}^{d}\right), G(x)=\left\|\hat{\varphi}_{\| x}\right\|_{\ell^{2}\left(\mathbf{Z}^{d}\right)}^{2}$, and $m(x)$ is also a scalar. (10) becomes

$$
\sum_{\alpha \in Q} \operatorname{rank}\left\|\widehat{\varphi}_{\|(x+\alpha) / 2}\right\|_{\ell^{2}\left(\mathbf{Z}^{d}\right)}^{2}-\operatorname{rank} \sum_{\alpha \in Q}\left|m\left(\frac{x+\alpha}{2}\right)\right|^{2}\left\|\hat{\varphi}_{\|(x+\alpha) / 2}\right\|_{\ell^{2}\left(\mathbf{Z}^{d}\right)}^{2} \leqslant 2^{d}-1,
$$

where the rank of a scalar is the rank of the $1 \times 1$ matrix with the scalar entry. Notice that the left-hand side of the above inequality is less than or equal to $2^{d}$. Hence the frame multiresolution analysis admits a standard wavelet set if and only if the left-hand side is 
not $2^{d}$ almost everywhere. The condition, now, is equivalent to the condition that $E$ is of zero Lebesgue measure with

$$
E:=\left\{x \in \mathbb{T}^{d}: \widehat{\varphi}_{\|(x+\alpha) / 2} \neq 0 \text { for each } \alpha \in Q, \sum_{\alpha \in Q}\left|m\left(\frac{x+\alpha}{2}\right)\right|^{2}=0\right\} .
$$

This recovers Theorem 5 of $[16]$ (see also $[2,14]$ for the univariate case).

We now observe a simple relationship between the spectrums of $V_{0}$ and $V_{1}$.

Lemma 8. $\sigma\left(V_{1}\right)=M^{*} \sigma\left(V_{0}\right)(\bmod 1)$.

Proof: Suppose that $x \in \sigma\left(V_{0}\right)$. Then $\widehat{\varphi}_{i \| x} \neq 0$ for some $i$ by (3). There exist $y \in \mathbb{T}^{d}, \alpha^{*} \in \mathbb{Z}^{d}$ such that $M^{*} x=y+\alpha^{*}$. Now $\alpha^{*}=\beta^{*}+M^{*} \gamma^{*}$ for some $\beta^{*} \in Q^{*}$ and $\gamma^{*} \in \mathbb{Z}^{d}$. (6) implies that

$$
\left\|a_{y, i, \beta^{*}}\right\|_{\ell^{2}\left(\mathbf{Z}^{d}\right)}=\left\|\widehat{\varphi}_{i \| M^{*-1}\left(M^{*} x-\beta^{*}-M^{*} \gamma^{*}+\beta^{*}\right)}\right\|_{\ell^{2}(\mathbf{Z})}=\left\|\widehat{\varphi}_{i \| x-\gamma^{*}}\right\|_{\ell^{2}} \neq 0 .
$$

Therefore, $y=M^{*} x-\alpha^{*} \in \sigma\left(V_{1}\right)$ by (7). This shows that $M^{*} \sigma\left(V_{0}\right) \subset \sigma\left(V_{1}\right)$. Suppose, on the other hand, that $x \in \sigma\left(V_{1}\right)$. Then $a_{x, i, \beta^{*}} \neq 0$ for some $1 \leqslant i \leqslant n$ and $\beta^{*} \in Q^{*}$ by (7). Then $\widehat{\varphi}_{i \| M^{\cdot-1}\left(x+\beta^{*}\right)} \neq 0$ by (6). Hence $M^{*-1}\left(x+\beta^{*}\right)(\bmod 1) \in \sigma\left(V_{0}\right)$ by (3). This shows that $\sigma\left(V_{1}\right) \subset M^{*} \sigma\left(V_{0}\right)(\bmod 1)$.

Recall that a frame is a Riesz basis if it is, in a certain sense, 'globally' irredundant, that is, irredundant in the norm topology $[9,13]$. Suppose that $\Phi$ is a quasi-stable generating set for $V_{0}$. Then the family of the integer translates of $\Phi$, which is a frame for $V_{0}$, is 'locally', that is, fibre-wise, irredundant. We now show that: if $\Phi$ and $\Pi$ are quasi-stable generating sets for $V_{0}$ and $V_{1}$, respectively, then the integer translates are 'globally' redundant. This result improves Theorem $3.9[15]$ in the sense that we do not presuppose that $\sigma\left(V_{0}\right)=\sigma\left(V_{1}\right)$. More precisely, we show:

THEOREM 9. Suppose that $\left\{V_{j}\right\}_{j \in \mathrm{Z}}$ is a frame multiresolution analysis. If $V_{0}$ and $V_{1}$ are both quasi-stable, and if $\operatorname{len} V_{1}=|\operatorname{det} M| \operatorname{len} V_{0}$, then they are actually stable. In particular, $\sigma\left(V_{0}\right)=\sigma\left(V_{1}\right)=\mathbb{T}^{d}$.

Proof: We may assume that $V_{0}=\mathcal{S}(\Phi)$ with $\Phi:=\left\{\varphi_{1}, \varphi_{2}, \ldots, \varphi_{n}\right\}$ a quasi-stable generator for $V_{0}$. Then $V_{1}=\mathcal{S}(\Pi)$ with $\Pi$ as in (2). Since \# $\#=\operatorname{len} V_{1}, \Pi$ is a generator for $V_{1}$ with minimal length. Hence $\Pi$ is actually a quasi-stable generator for $V_{1}$ [5, Theorem 3.12]. The length condition on $V_{1}$ and (8) show the following fact: If $x \in \sigma\left(V_{1}\right)$, then, for each $\alpha^{*} \in Q^{*}$, there exists $\delta_{x, \alpha^{*}} \in \mathbb{Z}^{d}$ such that $M^{*-1}\left(x+\alpha^{*}\right)+\delta_{x, \alpha^{*}} \in \sigma\left(V_{0}\right)$. It is obvious that for a fixed set of coset representatives $Q^{*}$ the set of 'folding' multi-integers $\left\{\delta_{x, \alpha^{*}}: x \in \sigma\left(V_{1}\right), \alpha^{*} \in Q\right\}$ is a finite set. This implies that $\sigma\left(V_{0}\right)$ contains a measurable subset of Lebesgue measure $\left|M^{*-1}\left(\sigma\left(V_{1}\right)+\alpha^{*}\right)\right|=\left|M^{*-1}\left(\sigma\left(V_{1}\right)\right)\right|$. We show that these subsets of $\sigma\left(V_{0}\right)$ do not overlap. Suppose that $M^{*-1}\left(x+\alpha^{*}\right)+\gamma=M^{*-1}\left(y+\beta^{*}\right)+\delta \in \mathbb{T}^{d}$ for $x, y \in \sigma\left(V_{1}\right), \alpha^{*}, \beta^{*} \in Q^{*}, \gamma, \delta \in \mathbb{Z}^{d}$. Then $x-y=\beta^{*}-\alpha^{*}+M^{*}(\delta-\gamma)$. Since the righthand side is an integer and since $x, y \in \mathbb{T}^{d}=[-1 / 2,1 / 2)^{d}, x-y=0$. Hence $\alpha^{*}=\beta^{*}$ and 
$\gamma=\delta$. Therefore $\sigma\left(V_{0}\right)$ contains \#Q* $=|\operatorname{det} M|$ number of subsets of Lebesgue measure $\left|M^{*-1}\left(\sigma\left(V_{1}\right)\right)\right|$. This shows that $\left|\sigma\left(V_{0}\right)\right| \geqslant\left|\sigma\left(V_{1}\right)\right|$. Since $V_{0} \subset V_{1}, \sigma\left(V_{0}\right) \subset \sigma\left(V_{1}\right)$. Consequently, $\sigma\left(V_{0}\right)=\sigma\left(V_{1}\right)$. Lemma 8 implies that $\sigma\left(V_{0}\right)=M^{*} \sigma\left(V_{0}\right)(\bmod 1)$. The ergodicity of the map $x \in \mathbb{T}^{d} \rightarrow M^{*} x(\bmod 1)$ in $\mathbb{T}^{d}$ implies that $\sigma\left(V_{0}\right)$ is either $\mathbb{T}^{d}$ or empty. Since it is not empty, it is $\mathbb{T}^{d}$.

The length condition in Theorem 9 is indispensable by Example 6 .

\section{REFERENCES}

[1] J.J. Benedetto and S. Li, 'The theory of multiresolution analysis frames and applications to filter banks', Appl. Comput. Harm. Anal. 5 (1998), 398-427.

[2] J.J. Benedetto and O.M. Treiber, 'Wavelet frames: multiresolution analysis and extension principle', in Wavelet transforms and time-frequency signal analysis, (L. Debnath, Editor) (Birkhaüser, Boston, 2000), pp. 3-36.

[3] C. de Boor, R. DeVore and A. Ron, 'On the construction of multivariate (pre)wavelets', Constr. Approx. 9 (1993), 123-166.

[4] C. de Boor, R. DeVore and A. Ron, 'Approximations from shift-invariant subspaces of $L^{2}\left(\mathbb{R}^{d}\right)$ ', Trans. Amer. Math. Soc. 341 (1994), 787-806.

[5] C. de Boor, R. DeVore and A. Ron, 'The structure of finitely generated shift-invariant spaces in $L_{2}\left(\mathbb{R}^{d}\right)^{\prime}, J$. Funct. Anal. 119 (1994), 37-78.

[6] M. Bownik, 'The structure of shift-invariant subspaces of $L^{2}\left(\mathbb{R}^{n}\right)$ ', J. Funct. Anal. 177 (2000), 282-309.

[7] O. Christensen, B. Deng and C. Heil, 'Density of Gabor frames', Appl. Comput. Harmon. Anal. 7 (1999), 292-304.

[8] K. Gröchenig and W. R. Madych, 'Multiresolution analysis, Haar bases, and self-similar tilings of $R^{n}$ ', IEEE Trans. Inform. Theory 38 (1992), 556-568.

[9] C. Heil and D. Walnut, 'Continuous and discrete wavelet transforms', SIAM Rev. 31 (1989), 628-666.

[10] H. Helson, Lectures on invariant subspaces (Academic Press, New York, 1964).

[11] R.-Q. Jia, 'Shift-invariant spaces and linear operator equations', Israel J. Math. 103 (1998), 258-288.

[12] R.-Q. Jia and C.A. Micchelli, 'Using the refinement equation for the construction of pre-wavelets, II: powers of two', in Curves and Surfaces, (P.J. Laurent, A. Le Méhauté and L. Schumaker, Editors) (Academic Press, New York), pp. 209-246.

[13] H.O. Kim and J.K. Lim, 'New characterizations of Riesz bases', Appl. Comput. Harmon. Anal. 4 (1997), 222-229.

[14] H.O. Kim and J.K. Lim, 'On frame wavelets associated with frame multi-resolution analysis', Appl. Comput. Harmon. Anal. 10 (2001), 61-70.

[15] H.O. Kim and J.K. Lim, 'Applications of shift-invariant space theory to some problems of multi-resolution analysis of $L^{2}\left(\mathbb{R}^{d}\right)$ ', in Wavelet Analysis and Applications, (D. Deng, D. Huang, R.-Q Jia, W. Lin and J. Wang, Editors) (American Mathematical Society/International Press, Boston, 2001), pp. 183-191.

[16] J.K Lim, 'Gramian analysis of multivariate frame multiresolution analyses', Bull. Austral. Math. Soc. 66 (2002), 291-300. 
[17] S. Mallat, 'Multiresolution approximations and wavelets', Trans. Amer. Math. Soc. 315 (1989), 69-88.

[18] Y. Meyer, Wavelets and operators, Cambridge Studies in Advanced Mathematics 37 (Cambridge University Press, Cambridge, 1992).

[19] A. Ron and Z. Shen,, 'Frames and stable bases for shift-invariant subspaces of $L_{2}\left(\mathbb{R}^{d}\right)$ ', Canad. J. Math. 47 (1995), 1051-1094.

[20] P. Walters, An introduction to ergodic theory, Graduate Texts in Mathematics 79 (Springer-Verlag, New-York, Berlin, 1981).

Division of Applied Mathematics

KAIST

373-1 Guseong-dong, Yuseong-gu

Daejeon 305-701

Korea

e-mail: hkim@amath.kaist.ac.kr rykim@amath.kaist.ac.kr

\section{CHiPS}

KAIST

373-1, Guseong-dong, Yuseong-gu

Daejeon, 305-701

Republic of Korea

e-mail: jaekun@ftn.kaist.ac.kr 